\title{
Measuring shear-induced self-diffusion in a counterrotating geometry
}

\author{
Victor Breedveld,* Dirk van den Ende, ${ }^{\dagger}$ Maarten Bosscher, Robert J. J. Jongschaap, ${ }^{\ddagger}$ \\ and Jorrit Mellema ${ }^{\S}$ \\ Rheology Group, Department of Applied Physics, Twente Institute of Mechanics, University of Twente, P.O. Box 217, \\ 7500 AE Enschede, The Netherlands \\ (Received 18 September 2000; published 23 January 2001)
}

\begin{abstract}
The novel correlation method to measure shear-induced self-diffusion in concentrated suspensions of noncolloidal hard spheres which we developed recently [J. Fluid Mech. 375, 297 (1998)] has been applied in a dedicated counterrotating geometry. The counterrotating nature of the setup enables experiments over a wider range of well-controlled dimensionless time $(\dot{\gamma} \Delta t$ in the range $0.03-3.5$, compared to $0.05-0.6$ in previous experiments; here $\dot{\gamma}$ denotes the shear rate and $\Delta t$ the correlation time). The accessible range of timescales made it possible to study the nature of the particle motion in a more detailed way. The wide radius geometry provides a well-defined flow field and was designed such that there is optical access from different directions. As a result, shear-induced self-diffusion coefficients could be determined as a function of particle volume fraction $\phi(0.20-0.50)$ in both the vorticity and velocity gradient direction. A transition could be observed to occur for $\dot{\gamma} \Delta t$ of $O(1)$, above which the particle motion is diffusive. The corresponding self-diffusion coefficients do not increase monotonically with particle volume fraction, as has been suggested by numerical calculations and theoretical modeling of Brady and Morris [J. Fluid Mech. 348, 103 (1997)]. After an exponential growth up to $\phi=0.35$, the diffusion coefficients level off. The experiments even suggest the existence of a maximum around $\phi=0.40$. The results are in good agreement with experimental literature data of Phan and Leighton [J. Fluid Mech. (submitted)], although these measurements were performed for much larger values of the dimensionless time $\dot{\gamma} \Delta t$.
\end{abstract}

DOI: 10.1103/PhysRevE.63.021403

PACS number(s): 83.80.Hj, 47.15.Pn, 47.55.Kf

\section{INTRODUCTION}

Shear-induced diffusion of noncolloidal particles is of great importance for understanding the transport processes in concentrated suspensions under flow and has therefore attracted a lot of attention [1].

The nature of the phenomenon is different from the more familiar diffusion concepts of Brownian diffusion in colloidal suspensions, which is caused by thermal fluctuations, and turbulent diffusion, driven by inertial effects. Shear-induced diffusion is the result of hydrodynamic interactions between suspended particles. In principle, this is a deterministic process. Due to the complex nature of the hydrodynamics, however, it can be described as a diffusion process. Shearinduced diffusion has been studied on two different levels: self-diffusion of individual particles and the collective process of gradient diffusion, which is studied by the net migration of particles on the macroscopic level [2]. In this paper we address self-diffusion. By studying the results of hydrodynamic interactions on the particle level, we want to acquire information about the microscopic processes behind shear-induced diffusion.

Only very few experimental studies on shear-induced self-diffusion have been reported up to now [3-5]. These measurements have all been performed by means of the same technique: a Couette cell is loaded with a concentrated sus-

\footnotetext{
*Email address: victor@engineering.ucsb.edu

${ }^{\dagger}$ Email address: H.T.M.vandenEnde@tn.utwente.nl

*Email address: R.J.J.Jongschaap@tn.utwente.nl

${ }^{\S}$ Email address: J.Mellema@tn.utwente.nl
}

pension of noncolloidal particles, to which a tagged tracer particle is added and after each full rotation in the Couette cell, the passage time of the tracer particle is detected. Fluctuations in the tracer position and its revolution time can be related to shear-induced self-diffusion coefficients.

The major disadvantage of the technique is the fact that observations are only possible after full revolutions in the Couette cell. Consequently, the effect can only be studied on relatively long times $\dot{\gamma} \Delta t \sim O$ (10) (the process is fully hydrodynamically driven so that the strain $\dot{\gamma} \Delta t$ is the appropriate dimensionless time, where $\dot{\gamma}$ denotes the shear rate and $\Delta t$ the passage time). Moreover, the values of $\dot{\gamma} \Delta t$ cannot be controlled externally, as is desirable when collecting detailed information about the nature of the particle displacements. The revolution time of the tracer particles in the Couette cell depends on their location at the start of the rotation and on the diffusive motion during the revolution: a particle close to the rotating outer wall of the Couette cell moves considerably faster than a tracer particle at the stagnant inner wall.

To overcome the problem of ill-controlled strain values $\dot{\gamma} \Delta t$ we have recently developed a correlation method and applied it successfully in a Couette cell with rotating inner cylinder [6]. The setup enabled accurate measurements over a controlled strain range from $\dot{\gamma} \Delta t=0.05$ to circa 0.60 .

In this paper, new results are presented, which have been obtained with the same technique, but in a more sophisticated counterrotating geometry. The new setup dramatically enlarges the experimentally accessible range of $\dot{\gamma} \Delta t$ (now $0.03-3.5)$, bridging the gap between our previous experimental results [6] and the literature data. In the next section the basic equations of the correlation technique are briefly presented. A description of the apparatus and materials fol- 


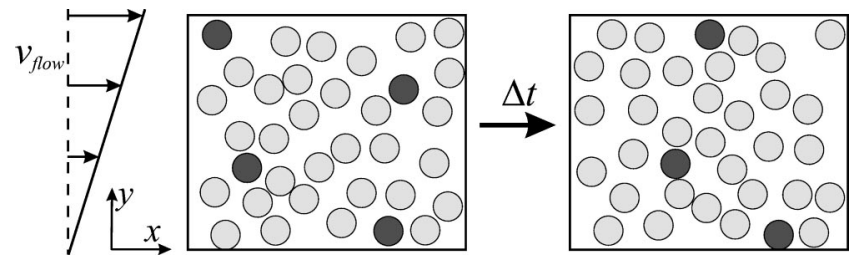

FIG. 1. Images of tracer particles in a concentrated suspension under shear.

lows in Sec. III. We present and discuss results in Sec. IV and finally conclusions are drawn.

\section{CORRELATION TECHNIQUE}

This section contains a brief review of the basic concepts of the novel correlation technique. For a detailed mathematical description we refer to a previous paper [6].

The experimental technique is based on spatial correlation of a sequence of video images of the sheared suspension. The majority of particles is refractive index matched with the suspending fluid and only a small fraction of colored tracer particles is used, so that even concentrated suspensions (20$50 \%$ volume fraction of particles) are optically accessible and images can be taken with a digital CCD camera at a fixed position in the geometry. Due to the refractive index matching in these images only the tracer particles show up and two consecutive images appear like the pictures in Fig. 1. The macroscopic flow field is drawn on the left. In accordance with common conventions, $x$ denotes the velocity direction, $y$ the velocity gradient direction and $z$ the vorticity direction.

The positions of tracer particles in the images can be determined with state-of-the-art image analysis software. Subsequently, the positions of the tracer particles in the second image are correlated with the positions of the tracers in the first image by calculating the displacement vectors $(\Delta x, \Delta y)$.

Figure 2 shows the set of correlation vectors for the image pair of Fig. 1. At this point it is important to note that only the autocorrelation vectors (thick lines) contain information about self-diffusion, since they represent displacements of individual particles. The cross-correlation (dashed) vectors have no contribution to self-diffusion and only comprise information about the spatial distribution of tracer particles over the window of observation. The main problem is to discriminate between auto- and cross-correlation vectors

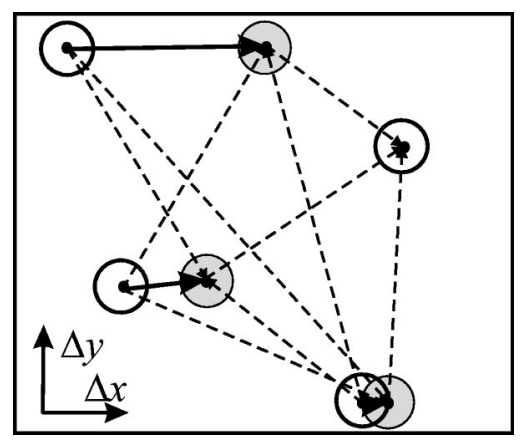

FIG. 2. Correlation vectors $(\Delta x, \Delta y)$ for the images of Fig. 1.

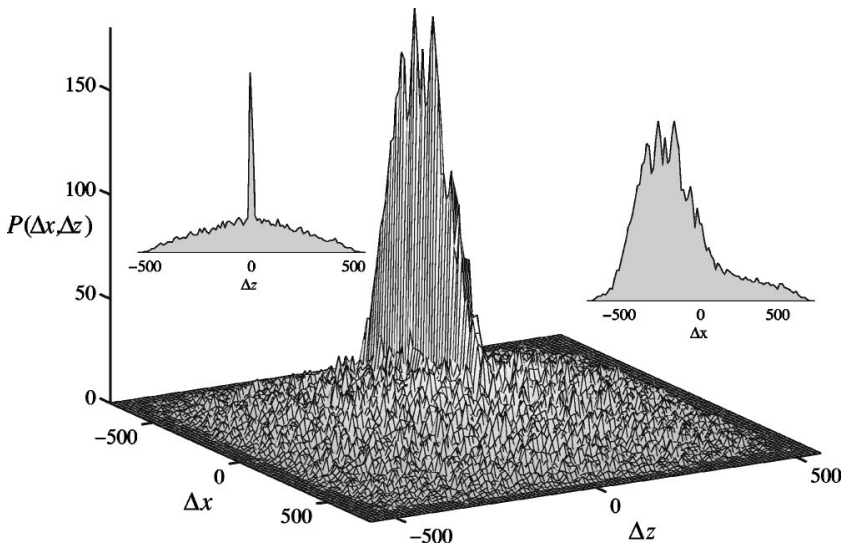

FIG. 3. Histogram of correlation vectors $(\Delta x, \Delta z)$ for an ensemble of 8000 images $(\dot{\gamma} \Delta t=0.72$ and $\phi=0.30)$; the units of the displacement axes $(\Delta x$ and $\Delta z)$ are pixels and the vertical axis represents the probability $P$ in arbitrary (non-normalized) units. The contours of the projections along both axes are shown as well.

with the objective to eliminate the cross-correlation contributions. As described in [6] this can be achieved statistically by determining the correlation vectors for a large ensemble of image pairs (at constant time interval $\Delta t$ ) and collecting all vectors in a histogram. A characteristic example is given in Fig. 3, representing data from an experiment in our counterrotating geometry. The camera is looking along the velocity gradient $(y)$ axis, so that the plane of view is the $x-z$ plane. Projections along the axes of the three-dimensional (3D) plot are included to accentuate the shape of the peak.

The peak in Fig. 3 consists of all autocorrelation vectors (thick lines in Fig. 2) which are relevant to this study, while the noisy background is due to the cross-correlation vectors (dashed). In other words, the peak contains the desired information about the motion of tracer particles. In particular its position represents the average displacement of tracers during the correlation time step $\Delta t$. This property is used in particle image velocimetry (PIV), a technique which is nowadays frequently employed in experimental fluid dynamics to measure the velocity profiles of complicated flow fields (see, e.g., [7,8]). In addition to this well-known feature, the width of the correlation peak is a measure for the size of fluctuations in the particle displacements during time step $\Delta t$, which are the signature of diffusion on the particle level. Hence the width of the correlation peak is the quantity of interest for studying shear-induced self-diffusion. This idea forms the basis of our correlation technique.

As emphasized by the contours of the projections in Fig. 3 , the peak width is strongly amplified in the $\Delta x$ direction. This is caused by the convective shear flow, because tracer particles on different stream lines experience different $\Delta x$ displacements in the shear flow. Such a convective contribution does not exist in the vorticity $(z)$ direction, where particle motion is purely the result of fluctuations. Neglecting the displacements in the $\Delta x$ direction, which are obscured by the convection, we can obtain accurate information about the $\Delta z$ fluctuations by summing the data along the $\Delta x$ axis. Such a summation procedure results in the histogram of Fig. 4 which represents the probability of encountering a certain $\Delta z$ 


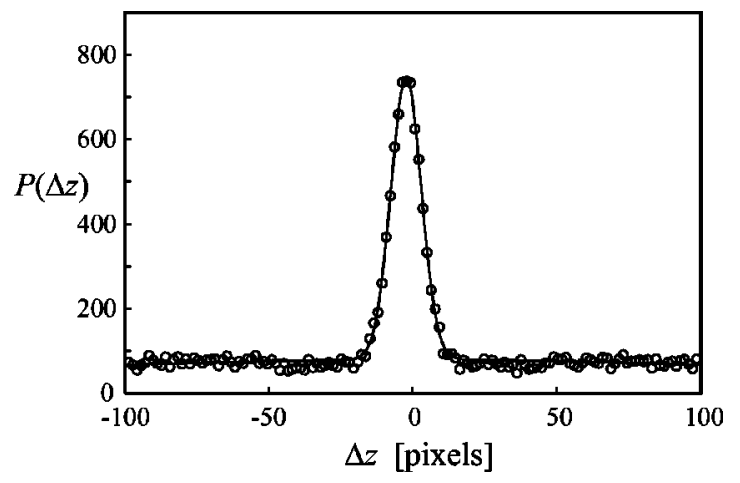

FIG. 4. Reduced histogram of Fig. 3, revealing the Gaussian nature of the particle displacements in the vorticity direction $(\Delta z)$ for an ensemble of 8000 images $(\dot{\gamma} \Delta t=0.72$ and $\phi=0.30)$; the curve shows the nonlinear fit of the data with eq. (1); units of the displacement axis $(\Delta z)$ are pixels (particle radius $a=31$ pixels) and the vertical axis represents the probability $P$ in arbitrary (nonnormalized) units.

value in the image ensemble. It was shown [6] that the peak in Fig. 4 should have a Gaussian shape in the $\Delta z$ direction, if the particle fluctuations behave diffusively (see Eqs. 2.15 and 3.3 of [6]):

$$
P(\Delta z)=\left(1-\left|\frac{\Delta z}{H}\right|\right)\left\{C+B \cdot \exp \left(-\frac{\Delta z^{2}}{2 \sigma_{z}^{2}}\right)\right\}
$$

with $H$ the size of the image in the $z$ direction and

$$
\sigma_{z}^{2}=2 D_{z z} \Delta t
$$

$D_{z z}$ being the diffusion coefficient in the vorticity direction. $B$ and $C$ both are functions that depend on the time step $\Delta t$ and on experimental parameters like image size, depth of focus, tracer concentration and particle detection efficiency of the image analysis procedure. For fixed experimental conditions, $B$ has a constant value. $C$ on the other hand represents the cross-correlation contribution to the histogram and is a function of the distribution of the tracer particles. To be more specific, $C$ depends on $P_{2}(\Delta \mathbf{x} ; \Delta t)$, the chance of finding a particle at a certain position $\mathbf{x}+\Delta \mathbf{x}$, while another particle resided at any possible position $\mathbf{x}$ a time $\Delta t$ earlier. For $\Delta t=0$, this function matches the well-known pair distribution function $g(\mathbf{r})$.

Because in general $g(\mathbf{r})$ can be expected to be nonuniform-particularly at high volume fractions where particles are not necessarily distributed randomly - the functional shape of $P_{2}(\Delta \mathbf{x} ; \Delta t)$ is a priori unknown. Therefore the exact theoretical shape of the curve in Fig. 4 is also uncertain.

For the noncounterrotating Couette setup this theoretical complication could be evaded. It was shown [6] that symmetry considerations are sufficient to eliminate the unknown function $C$. The key feature in the derivation is the fact that, because of the convective shear flow, the autocorrelation peak was always fully located in the half-plane $\Delta x>0$ of Fig. 3. Therefore the half-plane for $\Delta x<0$ only contained cross-correlation contributions, which could be subtracted from the points with $\Delta x>0$, using the flow symmetry. In this case Eq. (1) simplifies to

$$
P(\Delta z)=B\left(1-\left|\frac{\Delta z}{H}\right|\right) \exp \left(-\frac{\Delta z^{2}}{2 \sigma_{z}^{2}}\right) .
$$

When making use of the advantage of counterrotating flow, however, the plane of focus is located around the plane of zero velocity. Within the depth of focus of the camera optics, some of the tracer particles will experience flow displacements to the left and others will travel to the right. Consequently, the peak of Fig. 3 is located around the origin and symmetry arguments can no longer be used to subtract the cross-correlation contributions, since it would eliminate (part of) the desired autocorrelation peak as well. In this case, a more detailed analysis of the role of $P_{2}(\Delta \mathbf{x} ; \Delta t)$ is required.

The influence of $P_{2}(\Delta \mathbf{x} ; \Delta t)$ on the shape of function $C$ in Eq. (1) is weakened by an averaging integral (Eq. 2.15 of [6]) as result of the finite depth of focus of the camera optics. The two-dimensional image is in fact the projection of a three-dimensional volume element, its size being defined by the depth of focus. Thus any possible spatial structure in the particle distribution function $P_{2}$ is smeared out by the projection.

We have used a robust experimental analysis to clarify the effect of volume averaging on the observed shape of the cross-correlation contribution. To this purpose the functional shape of $C$ was determined for $\Delta t=0$, i.e., when $P_{2}(\Delta \mathbf{x} ; \Delta t)$ matches $g(\Delta \mathbf{x})$. The cross-correlation vectors between different particles in every image were calculated and the collected data were plotted in histograms like Figs. 3 and 4. These histograms showed that within experimental error no spatial structure could be detected. The distribution appeared to be random, most probably due to the volume averaging integral that represents projection. If no structure can be found for $g(\Delta \mathbf{x})$ at $\Delta t=0$, it is to be expected that this will be even less likely for $P_{2}(\Delta \mathbf{x} ; \Delta t)$ at finite values of $\Delta t$.

Consequently, in our counterrotating experiments we could safely apply the assumption of $C$ being constant, so that Eq. (1) could be used directly to fit the experimental data of histograms like Fig. 4 and determine the width of the autocorrelation peak.

The single relevant timescale of the experiment is $\dot{\gamma} \Delta t$ : the process stops when the flow is stopped and Brownian motion is negligible. Furthermore the only important length scale is the particle radius $a$. Thus, dimensional analysis requires that $\sigma_{z}$ in Eq. (2) obeys the following scaling [4,5]:

$$
\frac{\sigma_{z}^{2}}{a^{2}}=2 \hat{D}_{z z} \dot{\gamma} \Delta t
$$

where $\hat{D}_{z z}$ is the dimensionless diffusion coefficient, scaled by $\dot{\gamma} a^{2} . \hat{D}_{z z}$ can be expected to depend on the particle volume fraction $\phi$. These simple scaling arguments have been confirmed by several experimental studies $[3,5,6]$. One of the most remarkable differences between shear-induced diffu- 
sion and Brownian diffusion is the fact that the former is independent of the viscosity of the suspending fluid.

The correlation technique can be used to investigate the nature of the particle motion over a range of $\dot{\gamma} \Delta t$ values. In regions where the scaling of Eq. (4) applies, the motion is diffusive and the dimensionless diffusion coefficient $\hat{D}_{z z}$ can be calculated directly from the slope in a $\sigma^{2} / a^{2}-\dot{\gamma} \Delta t$ graph. This is a powerful feature of the method, since the selfdiffusion can thus be investigated on different timescales as opposed to the technique employed by Leighton and Acrivos [5] and Phan and Leighton [3] where single points after relatively long times were used to derive a diffusion coefficient without actually checking the diffusive scaling.

When the system is viewed from the vorticity $(z)$ direction, similar graphs are obtained and relations analogous to Eqs. (3) and (4) can be derived for the width of the autocorrelation peak in the $\Delta y$ direction, $\sigma_{y}$, and accordingly $\hat{D}_{y y}$ can be measured.

\section{EXPERIMENTAL WORK}

The technique described in the preceding section has been successfully applied and tested in a Couette geometry [6]. Due to geometrical restrictions, the range of $\dot{\gamma} \Delta t$ in those experiments was limited from 0.05 to 0.6 . For larger strain values, the shear flow moved too many particles out of the viewing window for obtaining reliable statistical information. In order to circumvent this problem and to increase the experimentally accessible range of $\dot{\gamma} \Delta t$, a counterrotating geometry was built, in which the images could be taken close to the plane of zero velocity. In this way particles were kept within the field of view for much longer times.

\section{A. Apparatus}

Our experimental setup was designed with a cone-plane geometry, since it is characterized by a constant shear rate even if the fluid is non-Newtonian. The value of the important scaling parameter $\dot{\gamma}$ could thus be controlled more accurately and collective migration as a result of gradient diffusion could be neglected. In literature, the importance of gradient diffusion in cone-plate and plate-plate geometries has been subject of debate for some time [9-11]. Conclusive evidence was not available at the moment of designing our apparatus. Presently, the argument seems to develop in favor of the existence of outward particle migration in cone-plate flow due to curvature effects $[11,12]$. However, significant particle migration could not be observed in our cone-plate geometry, possibly due to the small curvature of the wide radius design.

Figure 5 shows a sketch of the apparatus, which is an adapted version of the rheoscope that was developed to study the deformation of vesicles in shear flow [13]. The flow cell units were connected to independent drive units (step motors with RS100 control, designed and built by Eltromat) via precision fittings and play-free Oldham couplings. The units were further supported by aerostatic bearings so that extremely high accuracy could be achieved in the alignment of the flow cell.

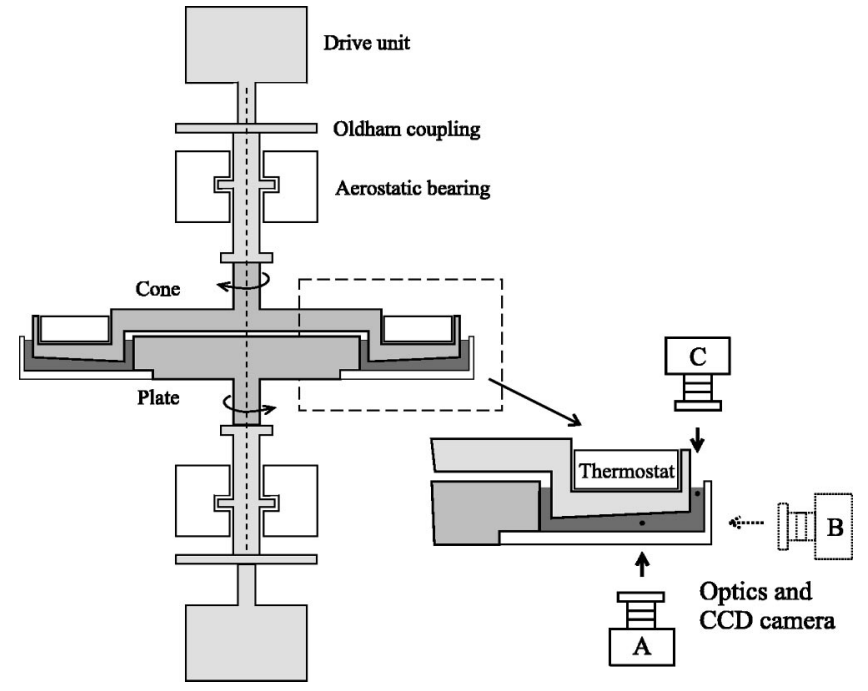

FIG. 5. Design of counterrotating cone-plate geometry.

In our experiments we made use of a steel cone and a glass plate for optical access from different directions. The cone-plate configuration (with the extrapolated cone intersecting the plate at the rotational axis) was only present over a limited range of the radius, from 8.5 to $11.5 \mathrm{~cm}$. This geometry was chosen in order to restrict the sample volume, while maintaining a small cone angle $\theta=2^{\circ}$ and a sufficiently large gap size to minimize wall effects for noncolloidal particles of $O(100 \mu \mathrm{m})(3.0 \mathrm{~mm}$ at the inner edge and $4.0 \mathrm{~mm}$ at the outer edge of the truncated cone). The cone and plate were both polished with extreme care, so that vertical variations during a full rotation were only about $5 \mu \mathrm{m}$ for both cone and plate. The temperature was controlled with a thermostat unit, which was brought into contact with the rotating steel cone through a thin water layer.

Numerical calculations were performed to check the influence of the cone edges on the steady state flow field in the tangential direction. The calculations led to an adaptation in the design: the inner section of the cone unit was made flat in order to minimize the influence of the sharp inner edge. It was shown that with this layout the flow was not significantly affected for both Newtonian and power-law fluids. The shear rate $\dot{\gamma}$ under the center of the cone deviated no more than a few tenths of a percent from the desired coneplate value.

The original idea was to have visual access to an observation window under the cone from two directions, so that all components of the diffusion tensor could be measured under exactly the same flow conditions. However, it turned out that measurements from position B in Fig. 5 were impossible. Despite extensive attempts to optimize the refractive index matching of our suspensions, the optical penetration depth could not be increased to more than $3 \mathrm{~mm}$, while about $7 \mathrm{~mm}$ would have been necessary for reliable measurements from position B (see also Sec. III B). An alternative approach was therefore taken to measure diffusion in the $x-y$ plane: the cone was lowered toward the glass plate and the outer Couette gap between cone and plate element was used for experiments, with camera position $\mathrm{C}$. The wide-radius Cou- 
ette gap has inner and outer radii $R_{i}=117.3 \mathrm{~mm}$ and $R_{o}$ $=121.6 \mathrm{~mm}$. The height of the fluid in the gap was chosen to be $8 \mathrm{~mm}$, so that the influence of the cone-plate section on the velocity profile was minimized, which was confirmed by numerical calculations. The mechanical precision of the flow elements was very high in the outer gap as well, the variations in gap width being no larger than $10 \mu \mathrm{m}$ during a full rotation. In essence we have used the same geometry for the measurements of $\hat{D}_{y y}$ as described in [6], but the counterrotating character of the apparatus dramatically increases the maximum correlation time.

The conversion from rotational speeds to shear rates is based on standard equations for cone-plate and Couette rheometry:

$$
\begin{gathered}
\dot{\gamma}_{A}=\frac{2 \pi}{60} \frac{\Omega}{\theta}=3.0 \Omega, \\
\dot{\gamma}_{C}=\frac{2 \pi}{60} \frac{R_{o}^{2}+R_{i}^{2}}{R_{o}^{2}-R_{i}^{2}} \Omega=2.89 \Omega,
\end{gathered}
$$

the indices indicating the viewing position and $\Omega[\mathrm{rpm}]$ being the relative rotational speed of cone and plate. For the Couette system, Eq. (6) represents the shear rate $\dot{\gamma}_{C}$ at the position in the gap where the tangential velocity equals zero, since the images are collected at this location:

$$
r=\left(\frac{2 R_{o}^{2} R_{i}^{2}}{R_{o}^{2}+R_{i}^{2}}\right)^{1 / 2}
$$

The drive units are capable of producing shear rates ranging from $3 \times 10^{-3}$ to $30 \mathrm{~s}^{-1}$, but all experiments were carried out for $\dot{\gamma}$ between 0.1 and $2.0 \mathrm{~s}^{-1}$.

Since both the initial positions and displacements of the tracer particles are available from image analysis and correlation procedures, for the Couette geometry (observation window in the $x$ - $y$ plane) it is possible to determine the velocity field of the macroscopic shear flow experimentally from the data. The measured values were higher than the Couette predictions of Eq. (6) for Newtonian fluids. For the study presented in this paper, we have used the experimental values for the shear rate, since they accurately represent the actual shear rate at the observation location in the gap. The procedure to measure the velocity field is explained and discussed in detail in [14].

\section{B. Materials}

Experiments were performed with suspensions of PMMA (polymethylmethacrylate) particles (produced by ICI, class 4F) dispersed in a fluid consisting of demineralized water (9.2 wt.\%), zinc-II-chloride (13.4\%), and Triton X-100 (77.4\%), following the recipe of [11]. The fluid was Newtonian with a viscosity of $3.4 \mathrm{~Pa} \mathrm{~s}$ at $23^{\circ} \mathrm{C}$ and matched both the density $(1.172 \mathrm{~kg} / \mathrm{l})$ and refractive index $\left(n_{D}^{25}=1.491\right)$ of the particles. In our attempts to measure from camera position B (Fig. 5), we have tried to improve the refractive index matching by changing the fluid composition. However, we never obtained penetration depths larger than $3 \mathrm{~mm}$. Most likely this is due to small variations in refractive index between different particles or even within particles. We have noticed that changes in the fourth decimal of the refractive index are sufficient to cause significant blurring effects of the video images. The particles were sieved repeatedly to obtain fairly monodisperse particles with a diameter of 90 $\pm 15 \mu \mathrm{m}$. Spheres with air bubbles were removed by means of density segregation. The particles were suspended in a water-glycerol mixture of a slightly lower density and the creaming fraction of light particles was removed. A small fraction of the particles, typically 0.2 vol. $\%$ of the suspension, was colored with liquid fabric dye (RIT) to serve as tracer particles in the transparent suspension; no changes could be found in their density after coloring. The suspensions were homogenized by gently tumbling the components overnight at low rotational speed. Then they were set to rest to let the small quantity of air bubbles disappear. Measurements were performed for particle volume fractions $\phi$ of $20-50 \%$.

\section{Image acquisition and analysis}

As shown in Fig. 5 the transparent plate provided optical access to the suspension volume from position A, which was used to measure $\hat{D}_{z z}$. From position $\mathrm{C}$ we entered the suspension through the free surface in order to find $\hat{D}_{y y}$. A digital CCD camera (JAI M-10, 768 by 582 pixels, 25 images per second) was mounted with optics to obtain a view window of 1.10 by $0.83 \mathrm{~mm}$ (determined by calibration) and connected to a PC. Illumination was arranged with a fiber optic halogen illuminator (Schott KL1500) that provided optimal contrast in the images.

The video images were stored directly on the PC through a frame grabber (Matrox Pulsar) that was operated using a special software tool (Hispa), which enabled accurate and controlled image acquisition of series of up to 200 images at discrete time intervals $\Delta t$ of $40 \mathrm{~ms}$ (video rate) and multiples thereof. Since Hispa offered the possibility of automatical repetition of the acquisition procedure, the total number of images in a run was limited only by the hardware storage capacity of the computer system. In our case, runs were made of up to 8000 images.

All images were then analyzed with a commercial software package for image analysis (Optimas). Basic analysis tools provided the means to identify dark objects and extract information about their properties. These properties-size, sphericity and blackness-were compared to preset criteria in order to decide whether the object was a tracer particle. If so, its position (gray value weighted center of mass) was stored in a data file for correlation purposes. The accuracy of the image analysis procedures was checked by changing the settings of the different analysis steps and monitoring the result. The error in the particle positions was estimated to be circa 0.2 pixels under our experimental conditions. The subpixel accuracy is the result of averaging over many pixels.

\section{RESULTS AND DISCUSSION}

The data files with particle positions were used as input for the correlation procedure. As described in Sec. II (see 

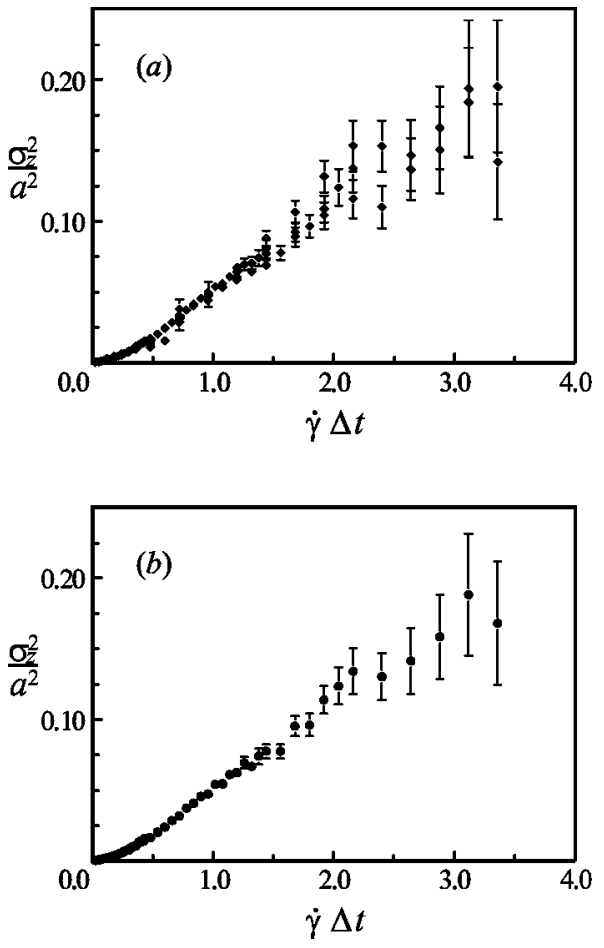

FIG. 6. Scaling of the average squared displacement of the particles $\sigma_{z}^{2} / a^{2}$ with dimensionless time $\dot{\gamma} \Delta t$ for $\phi=0.30$; (a) contains the raw fit data and (b) the results of the weighted average.

also [6] for detailed description) the correlation vectors were calculated for all possible image combinations and grouped according to the correlation time $\dot{\gamma} \Delta t$ between images.

\section{A. Correlation results}

The resulting sets of correlation vectors for all values of $\dot{\gamma} \Delta t$ were collected in histograms (e.g., Fig. 4), which were fitted with a nonlinear least square method to Eq. (1). The peak width $\sigma_{z}$ obtained from the fitting was plotted against the strain $\dot{\gamma} \Delta t$ in a $\sigma_{z}^{2} / a^{2}-\dot{\gamma} \Delta t$ graph in order to check the validity of scaling Eq. (4).

At each particle volume fraction, this procedure was repeated for different values of $\dot{\gamma}$ and $\Delta t$, thus covering the entire range of $\dot{\gamma} \Delta t$ between 0.03 and 3.5. Figure 6(a) shows the entire collection of data points for $\phi=0.30$. The error bars of the individual points represent the errors of the nonlinear fit. These statistical error estimates represent the scatter between different experimental runs very well. Since for most $\dot{\gamma} \Delta t$ values several data points were collected at different shear rates $\dot{\gamma}$, standard statistical manipulation of the data was appropriate. Figure 6(b) shows the resulting weighted averages (with $1 /$ error $^{2}$ as weight factor) and their associated standard deviations for $\phi=0.30$.

The figure displays a number of interesting features. It distinctly shows that for large values of $\dot{\gamma} \Delta t$ the statistical information becomes less accurate, since less and less tracer particles remain within the field of view during the entire correlation time. Although the counterrotating design strongly increased the range of accessible timescales and theoretically enables experiments at very large strain values,
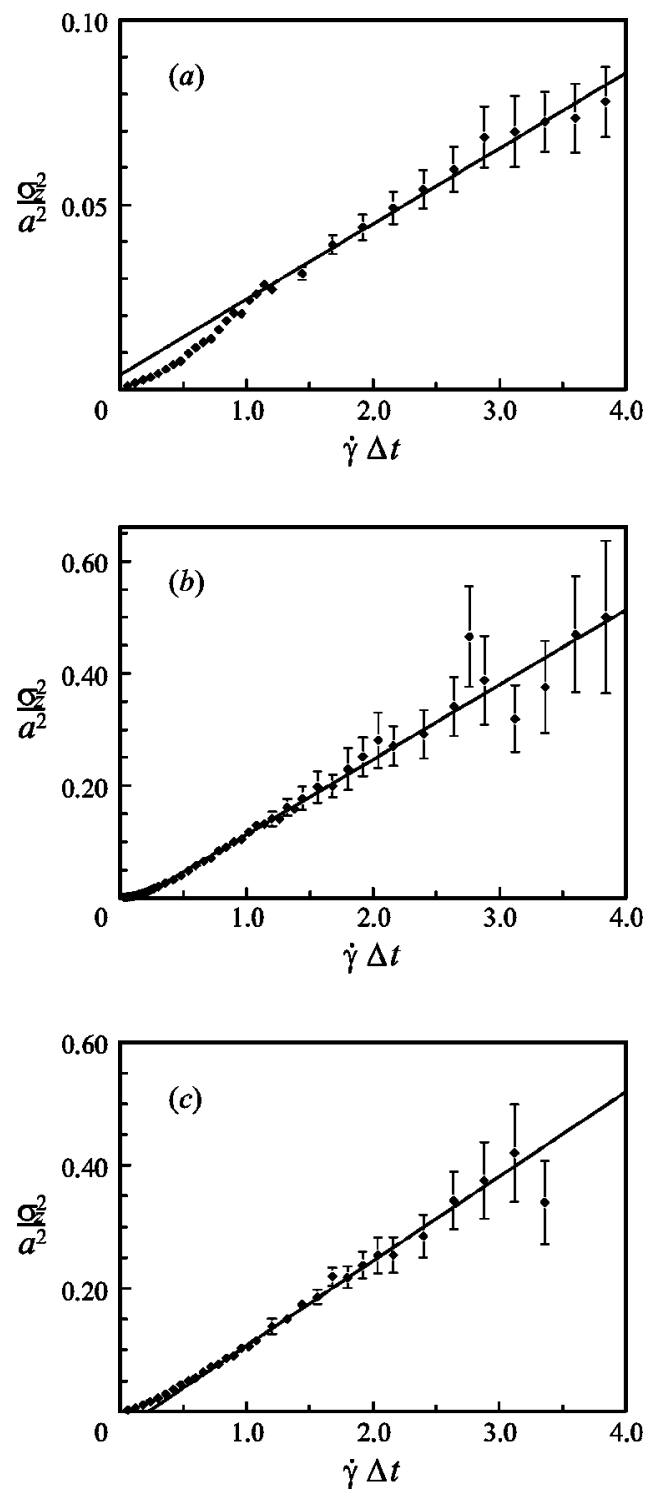

FIG. 7. Scaling of the average squared displacement of the particles $\sigma_{z}^{2} / a^{2}$ with dimensionless time $\dot{\gamma} \Delta t$ for different volume fractions (a) $\phi=0.20$, (b) $\phi=0.45$ and (c) $\phi=0.50$; the lines represent the linear fit to the data points at long times.

the $\dot{\gamma} \Delta t$ range was in practice limited by the number of images that could be stored and analyzed on the computer. In our case, the upper limit of $\dot{\gamma} \Delta t$ was circa 3.5. Increased hardware storage capacity and image analysis speed would extend the range of accessible strain values and decrease the error bars.

Figure 6(b) also shows linear scaling over most of the range, which is the fingerprint of diffusion [see Eq. (4)]. In this regime, roughly for $\dot{\gamma} \Delta t>0.7$, the data can be fitted linearly and the diffusion coefficient can be calculated directly from the slope of the fitted line, the dimensionless coefficient $\hat{D}_{z z}$ being half the slope. Figure 7 shows the experimental data in the vorticity direction and fitted line for various particle volume fractions.

Measurements in the velocity gradient $(y)$ direction were slightly more complicated, because the images had to be 

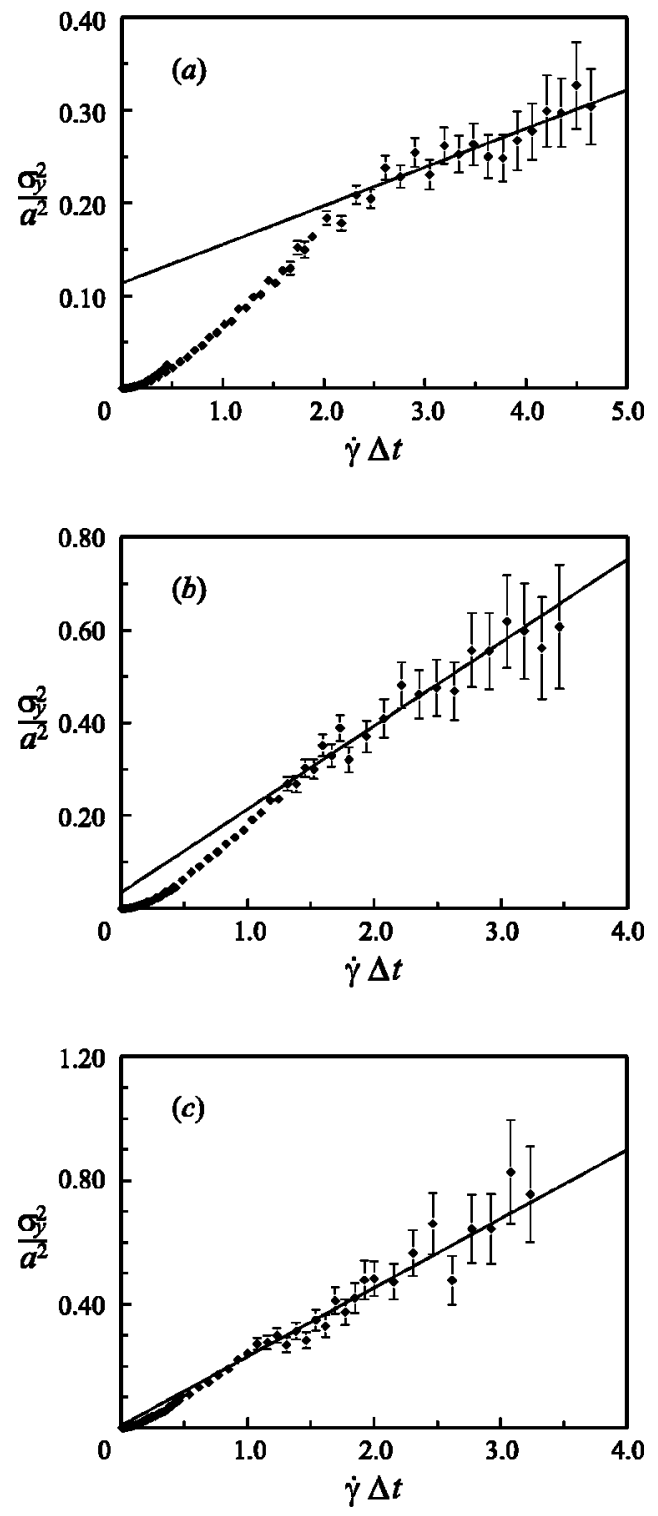

FIG. 8. Scaling of the average squared displacement of the particles $\sigma_{y}^{2} / a^{2}$ with dimensionless time $\dot{\gamma} \Delta t$ for different volume fractions (a) $\phi=0.20$, (b) $\phi=0.35$, and (c) $\phi=0.45$; the lines represent the linear fit to the data points at long times.

taken through the free surface in the Couette gap. In order to eliminate surface effects, the camera optics was focused on a location $1.5-2.0 \mathrm{~mm}$ below the surface. With a particle diameter of $100 \mu \mathrm{m}$ this should be sufficiently deep. In some experimental runs we deliberately focused on the particles at the suspension surface to investigate the surface effects and a significantly larger diffusion coefficient was found in those runs. In addition to the surface diffusion, measurements at the highest volume fractions are complicated by surface "roughening" as particles are pushed out of the suspension by strong hydrodynamic interaction forces. Thus the image quality deteriorates and the average number of detected tracer particles per image decreases, so that statistical information becomes less accurate. Nevertheless we have been able to obtain reliable results for diffusion in the $y$ direction. In Fig. 8 the scaling of the average squared displacement $\sigma_{y}^{2} / a^{2}$ is presented for different volume fractions together with the linear fit to the diffusive regime at long times. In comparison to Fig. 7, the onset of diffusion in the $y$ direction occurs for larger values of $\dot{\gamma} \Delta t$. The range of the linear fits has been adapted accordingly.

\section{B. Diffusion coefficients}

Within experimental errors the diffusion coefficient (slopes in Figs. 7 and 8) is constant down to $\dot{\gamma} \Delta t \sim 1$, which implies that the concept of shear-induced self-diffusion is applicable over a large range of timescales. In particular the lower boundary of validity is rather surprising. Within the framework of the collision model of Leighton and Acrivos [5] it was generally believed that diffusive behavior could only be attained on experimental times $\dot{\gamma} \Delta t \gg 1$, i.e., for times large compared to the assumed collision time in simple shear flow, so that a particle has experienced several interactions with its neighbors. The early onset of diffusion is the result of ensemble averaging. As soon as all particles have experienced a single displacement step, the correlations with previous moments in time are lost, since the direction of the displacement is random due to the random starting conditions of each interaction. As soon as the correlation has vanished, diffusive motion can be observed, although individual tracer particles have not yet undergone multiple displacements. The fact that characteristic time of the diffusion is of the order $\dot{\gamma} \Delta t=1$ does not therefore automatically imply that mechanistic (collision based) models are inadequate.

For small values of $\dot{\gamma} \Delta t$, the curves in Figs. 7 and 8 are nonlinear, so that diffusive motion cannot be assumed in this regime. Our previous experiments [6] were carried out for $\dot{\gamma} \Delta t$ varying from 0.05 to 0.6 , where in the current experiments linearity has not been reached. Within experimental accuracy of the former measurements it seemed appropriate to fit the data linearly, thus obtaining values for the diffusion coefficients. The present study suggests that in this way we have underestimated shear-induced self-diffusion. This is illustrated by Fig. 9. In the left graph, the data for $\phi=0.45$ of Fig. 7(c) are fitted linearly over two different ranges of $\dot{\gamma} \Delta t$. The dotted curve represents the linear fit for $\dot{\gamma} \Delta t$ between 0.1 to 0.6 , consistent with the procedures in [6], the solid curve depicts the linear fit only over long times, $\dot{\gamma} \Delta t>0.7$, analogous to Fig. 7.

In Fig. 9(b) the results of short-time linear fits of our current data in the $z$ direction are compared to the diffusion coefficients reported in [6] as a function of particle volume fraction $\phi$. Leaving out the data for $20 \%$, which were not very reliable, the figure shows that the previous study indeed underestimated the diffusion coefficient by up to $30 \%$ at the highest volume fractions. At the time of that study, we noticed deviations from the literature data of Phan and Leighton [3], but the differences were not very large in comparison with the significant experimental errors and the scatter in literature data. With our new results in mind, however, we must conclude that the accessible range of timescales in the original Couette experiments [6] was insufficient to capture the onset of shear-induced self-diffusion. 

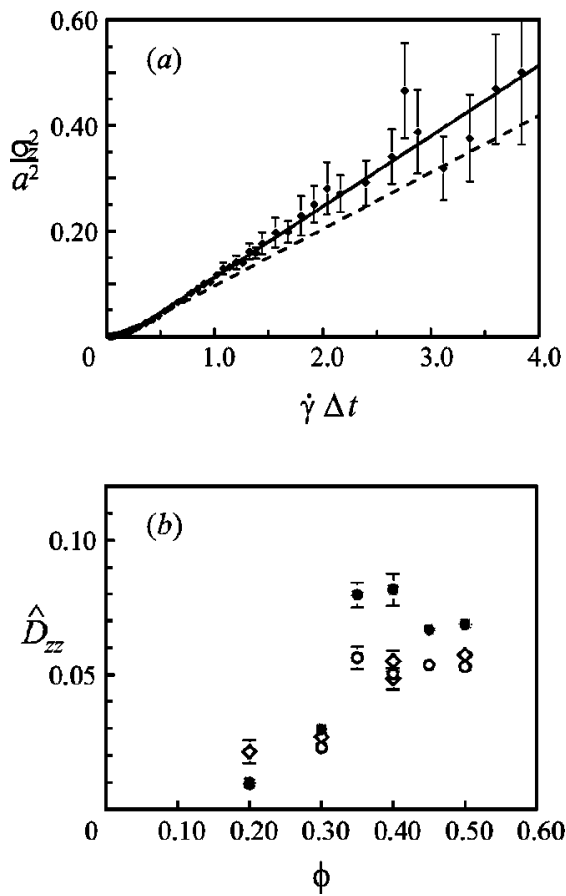

FIG. 9. Comparison of the data of [6] to the results of the current study. (a) shows two linear fits for $\phi=0.45$, dashed line (---) for $\dot{\gamma} \Delta t=0.10-0.60$, solid line (-) for $\dot{\gamma} \Delta t>0.7$. (b) contains the associated diffusion coefficients in the vorticity direction as a function of $\phi ;(\bigcirc)$ and $(\bigcirc)$ represent data from this study of, respectively, the full and limited fit, $(\diamond)$ denote earlier results of [6].

All available experimental data for shear-induced selfdiffusion as a function of volume fraction $\phi$ are combined in Fig. 10. The earlier results [6] were left out for the reason explained above. Even if the experimental errors are significant, we can safely say that there is fair agreement between the results of the various experimental methods with different systems. In particular our results are in very good agreement with the most recent data of other researchers, i.e., [3]. The results of Leighton and Acrivos [5] deviate somewhat from the other two studies, but in that study the authors had problems with crystallization of the suspending fluid and this could have affected their results.

The characteristic timescale $\dot{\gamma} \Delta t \sim 1$ above which we determine diffusion coefficients-and which was also used in [15] — has recently been questioned [16]. In their Stokesian dynamics simulations these authors found that linear (diffusive) scaling could only be observed for $\dot{\gamma} \Delta t>5$ in the reported cases. Unfortunately, even in our counterrotating setup we are unable to check this claim experimentally, because too many tracer particles leave the observation window at long times. However, we can compare our data to the results of Phan and Leighton [3], who performed their measurements over even longer times $(\dot{\gamma} \Delta t \gg 10$, although exact numbers cannot be reconstructed) and calculated the diffusion coefficient by assuming a straight line through the origin in graphs like our Fig. 7. On these long times the offset can indeed be neglected without problem. The agreement between our data and the long-time experiments is very suggestive: it is hard to understand that the slope of our linear fit
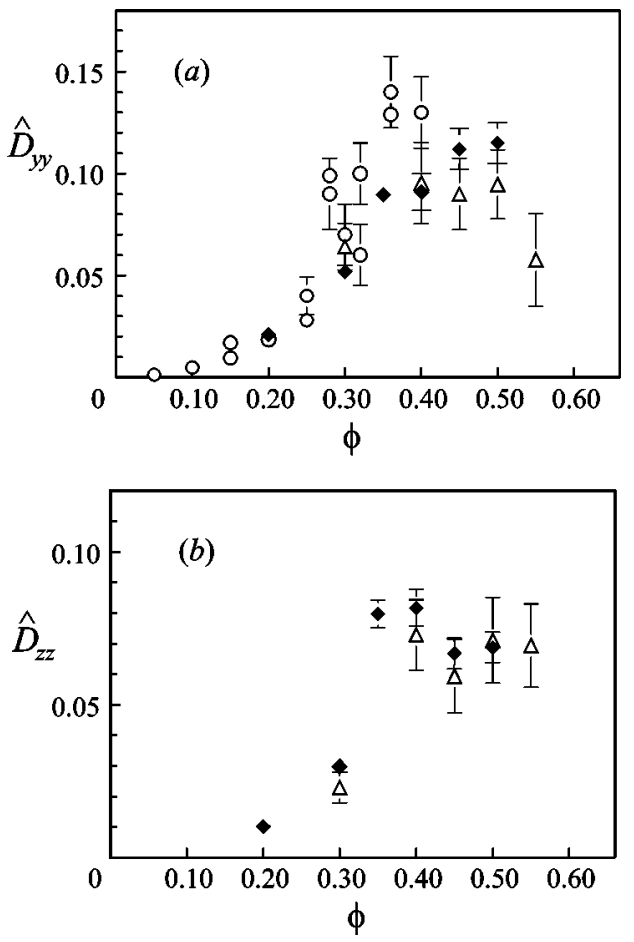

FIG. 10. Coefficients of shear-induced self-diffusion as measured by various researchers in (a) the velocity gradient $\hat{D}_{y y}$ and (b) the vorticity $\hat{D}_{z z}$ direction as a function of particle volume fraction $\phi ;(\triangle)$ represent data of [3], $(\bigcirc)$ of [7], and $(\diamond)$ were measured in this study.

could be incorrect, while extrapolation of this line accurately intersects the data points of [3].

Figure 10 reveals some intriguing trends. First of all the dimensionless diffusion coefficient in the velocity gradient direction $\left(\hat{D}_{y y}\right)$ is about a factor 1.5 larger than in the vorticity direction $\left(\hat{D}_{z z}\right)$. This is in agreement with the theoretical results of Brady and Morris [17], who predict shearinduced self-diffusion coefficients in concentrated suspensions by analyzing the microstructure of the suspension, i.e., the contact values of the pair distribution function $g(\mathbf{r})$. On the other hand the experimentally found anisotropy $\hat{D}_{y y} / \hat{D}_{z z}$ for concentrated suspensions is significantly smaller than the results of theoretical models for dilute suspensions that attribute shear-induced diffusion to the symmetry breaking of particle collisions due to particle roughness [18] or to the presence of a third particle [19]. Both models predict the anisotropy to be circa 10 and the discrepancy implies that the behavior of concentrated suspensions deviates strongly from the dilute limit.

Second, in Fig. 10 there is clear experimental evidence that both diffusion coefficients do not grow monotonically with volume fraction, as has been suggested by previous measurements [5] and by theoretical work [17]. Up to volume fractions of circa 0.35 the diffusion coefficients increase rapidly. Above this volume fraction they level off and even exhibit a tendency to go down for the highest volume fractions of 0.5 and 0.55 , although this final decrease cannot be concluded beyond experimental doubt. The measured diffu- 
sion coefficient $\hat{D}_{y y}$ at $45 \%$ lies somewhat higher than the trend in our experiments suggests and underlines the decrease in diffusivity at $50 \%$.

In their theoretical study Brady and Morris [17] have argued that shear-induced self-diffusion should scale as the product of the velocity fluctuations $(\dot{\gamma} a)$ and the size of the displacements $[O(a)]$. Since in their model the frequency of particle collisions is directly associated with the pair distribution function at contact, it should increase strongly with volume fraction, thus giving rise to growing self-diffusion coefficients as well. Our experimental results, however, reveal a different qualitative behavior. We have no explanation for this difference, but apparently one of the above scaling arguments is oversimplified. It could very well be that the contact value of the pair distribution function, $g(2)$, is insufficient to describe the hydrodynamic influence of the many particle problem for modeling shear-induced diffusion, although it has proven its usefulness in modeling suspension viscosities.

Stokesian dynamics simulations [20] have generally supported the theoretical ideas. However, recent numerical calculations of Foss and Brady [15] and Marchioro and Acrivos [16] have shown a tendency for $\hat{D}_{y y}$ to level off at high concentrations, even if it is hard to draw strong conclusions on the basis of their work, especially since $\hat{D}_{z z}$ does not exhibit the same qualitative behavior. The diffusion in the vorticity direction clearly increases with $\phi$ in both studies.

\section{CONCLUSIONS}

In combination with a counterrotating flow geometry the correlation technique [6] has proven to be a powerful method to study shear-induced self-diffusion of concentrated noncolloidal suspensions. Visual observation of tracer particle positions in a refractive index matched suspension at wellcontrolled time intervals enabled a detailed analysis of the particle motion. Statistical tools could be applied to accurately characterize this motion. The most important feature of the method is the fact that in the counterrotating coneplate geometry used in this study the dimensionless experimental time $\dot{\gamma} \Delta t$ could be varied from circa 0.03 to 3.5 , so that different timescales could be probed. The upper boundary of the $\dot{\gamma} \Delta t$ range is only limited by computer capacity for image storage and analysis.
The onset of self-diffusion could be observed and the associated long-time diffusion coefficients were determined as a function of particle volume fraction. The dimensionless diffusion coefficients $\hat{D}_{y y}$ and $\hat{D}_{z z}$ were in good agreement with previous results $[3,5]$ that were obtained with a different technique based on the analysis of the motion of single tracer particles over very long times $[\dot{\gamma} \Delta t \sim O(10)]$. The results significantly differed from our previous results in noncounterrotating Couette flow [1], but these discrepancies could be fully attributed to the limited range of $\dot{\gamma} \Delta t$ in those experiments $(0.05-0.60)$.

The onset of diffusion occurs at a dimensionless time $\dot{\gamma} \Delta t$ of $O(1)$, as could be expected, since $1 / \dot{\gamma}$ is the governing timescale in shear flow. The onset changes with particle volume fraction, shifting to shorter times at higher concentrations. In the velocity gradient direction, the diffusive regime is reached for larger strain values than in the vorticity direction. Recent numerical results [16] suggest that much longer times are needed to reach diffusive motion. Although we are unable to prove otherwise in our setup, the agreement with the measurements of Phan and Leighton [3] would be very peculiar if the regime of the linear fit in our experimental data is incorrect.

The measurements provide additional experimental evidence about the remarkable behavior of the diffusion coefficients as a function of particle volume fraction $\phi$. In contrast to recent theoretical predictions of Brady and Morris [17] and numerical results of Foss and Brady [15] and Marchioro and Acrivos [16], experimental self-diffusion coefficients in the velocity gradient and vorticity direction, respectively $\hat{D}_{y y}$ and $\hat{D}_{z z}$, do not increase monotonically as a function of $\phi$. Up to volume fractions of $35 \%$ the diffusion rises strongly and then it levels off and even shows the tendency to go down again above $45 \%$.

Furthermore, the experimentally determined values of the diffusion coefficient in the velocity gradient direction $\left(\hat{D}_{y y}\right)$ are significantly larger than in the vorticity direction $\left(\hat{D}_{z z}\right)$, the anisotropy being circa a factor 1.5 .

\section{ACKNOWLEDGMENT}

The research described in this paper was supported by the Foundation for Fundamental Research on Matter (FOM) in the Netherlands.
[1] R. Davis, J. Fluid Mech. 310, 325 (1996).

[2] A. Acrivos, J. Rheol. 39, 813 (1995).

[3] S. Phan and D. Leighton, J. Fluid Mech. (to be published).

[4] E. Eckstein, D. Bailey, and A. Shapiro, J. Fluid Mech. 79, 191 (1977).

[5] D. Leighton and A. Acrivos, J. Fluid Mech 177, 109 (1987a).

[6] V. Breedveld, D. van den Ende, A. Tripathi, and A. Acrivos, J. Fluid Mech. 375, 297 (1998).

[7] J. Westerweel, Ph.D. thesis, Delft Technical University, 1993 (unpublished).

[8] R. Adrian, Annu. Rev. Fluid Mech. 23, 261 (1991).
[9] B. Chapman and D. Leighton, Int. J. Multiphase Flow 17, 469 (1991).

[10] A. Chow, S. Sinton, J. Iwamiya, and T. Stephens, Phys. Fluids 6, 2561 (1994).

[11] G. Krishnan, S. Beimfohr, and D. Leighton, J. Fluid Mech. 321, 371 (1996).

[12] J. Morris and F. Boulay, J. Rheol. 43, 1213 (1999).

[13] K. de Haas, D. van den Ende, C. Blom, E. Altena, G. Beukema, and J. Mellema, Rev. Sci. Instrum. 69, 1391 (1998).

[14] V. Breedveld, Ph.D. thesis, University of Twente, 2000 (unpublished). 
[15] D. Foss and J. Brady, J. Fluid Mech. 401, 243 (1999).

[16] M. Marchioro and A. Acrivos, J. Fluid Mech. (to be published).

[17] J. Brady and J. Morris, J. Fluid Mech. 348, 103 (1997).

[18] F. da Cunha and E. Hinch, J. Fluid Mech. 309, 211 (1996).
[19] Y. Wang, R. Mauri, and A. Acrivos, J. Fluid Mech. 327, 255 (1996).

[20] Y. Yurkovetsky, Ph.D. thesis, Californian Institute of Technology, 1998 (unpublished). 